\title{
Deal or No Deal? End-to-End Learning for Negotiation Dialogues
}

\author{
Mike Lewis $^{1}$, Denis Yarats ${ }^{1}$, Yann N. Dauphin ${ }^{1}$, Devi Parikh ${ }^{2,1}$ and Dhruv Batra ${ }^{2,1}$ \\ ${ }^{1}$ Facebook AI Research $\quad{ }^{2}$ Georgia Institute of Technology \\ $\{$ mikelewis, denisy, ynd $@$ fb.com \{parikh, dbatra\}@gatech.edu
}

\begin{abstract}
Much of human dialogue occurs in semicooperative settings, where agents with different goals attempt to agree on common decisions. Negotiations require complex communication and reasoning skills, but success is easy to measure, making this an interesting task for AI. We gather a large dataset of human-human negotiations on a multi-issue bargaining task, where agents who cannot observe each other's reward functions must reach an agreement (or a deal) via natural language dialogue. For the first time, we show it is possible to train end-to-end models for negotiation, which must learn both linguistic and reasoning skills with no annotated dialogue states. We also introduce dialogue rollouts, in which the model plans ahead by simulating possible complete continuations of the conversation, and find that this technique dramatically improves performance. Our code and dataset are publicly available. ${ }^{1}$
\end{abstract}

\section{Introduction}

Intelligent agents often need to cooperate with others who have different goals, and typically use natural language to agree on decisions. Negotiation is simultaneously a linguistic and a reasoning problem, in which an intent must be formulated and then verbally realised. Such dialogues contain both cooperative and adversarial elements, and require agents to understand, plan, and generate utterances to achieve their goals (Traum et al., 2008; Asher et al., 2012).

\footnotetext{
${ }^{1}$ https://github.com/facebookresearch/ end-to-end-negotiator
}

We collect the first large dataset of natural language negotiations between two people, and show that end-to-end neural models can be trained to negotiate by maximizing the likelihood of human actions. This approach is scalable and domainindependent, but does not model the strategic skills required for negotiating well. We further show that models can be improved by training and decoding to maximize reward instead of likelihood-by training with self-play reinforcement learning, and using rollouts to estimate the expected reward of utterances during decoding.

To study semi-cooperative dialogue, we gather a dataset of 5808 dialogues between humans on a negotiation task. Users were shown a set of items with a value for each, and asked to agree how to divide the items with another user who has a different, unseen, value function (Figure 1).

We first train recurrent neural networks to imitate human actions. We find that models trained to maximise the likelihood of human utterances can generate fluent language, but make comparatively poor negotiators, which are overly willing to compromise. We therefore explore two methods for improving the model's strategic reasoning skillsboth of which attempt to optimise for the agent's goals, rather than simply imitating humans:

Firstly, instead of training to optimise likelihood, we show that our agents can be considerably improved using self play, in which pre-trained models practice negotiating with each other in order to optimise performance. To avoid the models diverging from human language, we interleave reinforcement learning updates with supervised updates. For the first time, we show that end-toend dialogue agents trained using reinforcement learning outperform their supervised counterparts in negotiations with humans.

Secondly, we introduce a new form of planning for dialogue called dialogue rollouts, in which an 
Divide these objects between you and another Turker. Try hard to get as many points as you can!

Send a message now, or enter the agreed deal!

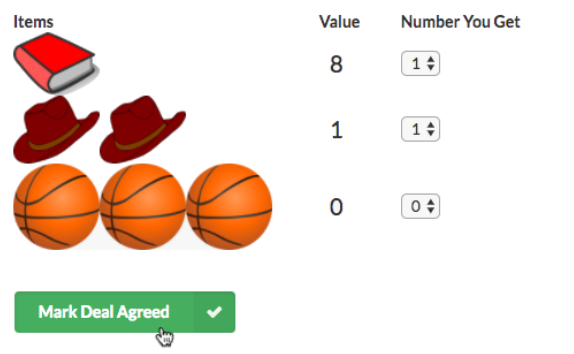

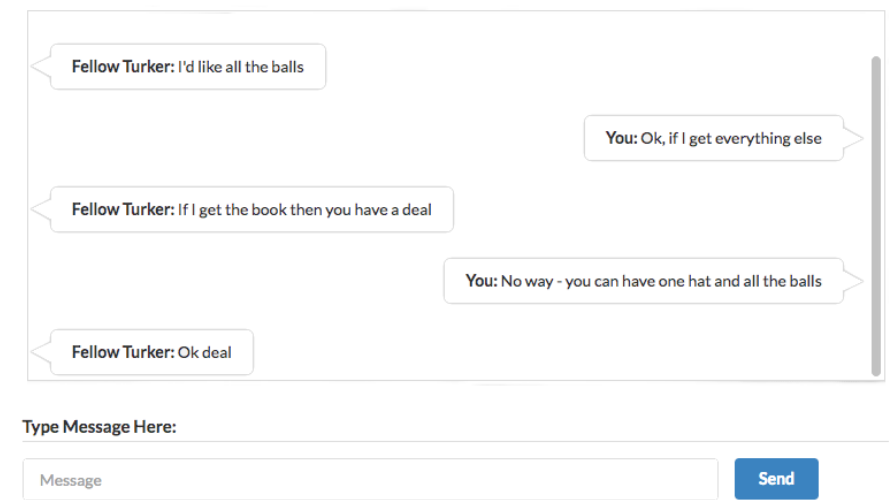

Figure 1: A dialogue in our Mechanical Turk interface, which we used to collect a negotiation dataset.

agent simulates complete dialogues during decoding to estimate the reward of utterances. We show that decoding to maximise the reward function (rather than likelihood) significantly improves performance against both humans and machines.

Analysing the performance of our agents, we find evidence of sophisticated negotiation strategies. For example, we find instances of the model feigning interest in a valueless issue, so that it can later 'compromise' by conceding it. Deceit is a complex skill that requires hypothesising the other agent's beliefs, and is learnt relatively late in child development (Talwar and Lee, 2002). Our agents have learnt to deceive without any explicit human design, simply by trying to achieve their goals.

The rest of the paper proceeds as follows: $\S 2 \mathrm{de}-$ scribes the collection of a large dataset of humanhuman negotiation dialogues. $\S 3$ describes a baseline supervised model, which we then show can be improved by goal-based training ( $(4)$ and decoding ( $\S 5)$. $\S 6$ measures the performance of our models and humans on this task, and $\S 7$ gives a detailed analysis and suggests future directions.

\section{Data Collection}

\subsection{Overview}

To enable end-to-end training of negotiation agents, we first develop a novel negotiation task and curate a dataset of human-human dialogues for this task. This task and dataset follow our proposed general framework for studying semicooperative dialogue. Initially, each agent is shown an input specifying a space of possible actions and a reward function which will score the outcome of the negotiation. Agents then sequentially take turns of either sending natural language messages, or selecting that a final decision has been reached. When one agent selects that an agreement has been made, both agents independently output what they think the agreed decision was. If conflicting decisions are made, both agents are given zero reward.

\subsection{Task}

Our task is an instance of multi issue bargaining (Fershtman, 1990), and is based on DeVault et al. (2015). Two agents are both shown the same collection of items, and instructed to divide them so that each item assigned to one agent.

Each agent is given a different randomly generated value function, which gives a non-negative value for each item. The value functions are constrained so that: (1) the total value for a user of all items is 10; (2) each item has non-zero value to at least one user; and (3) some items have nonzero value to both users. These constraints enforce that it is not possible for both agents to receive a maximum score, and that no item is worthless to both agents, so the negotiation will be competitive. After 10 turns, we allow agents the option to complete the negotiation with no agreement, which is worth 0 points to both users. We use 3 item types (books, hats, balls), and between 5 and 7 total items in the pool. Figure 1 shows our interface.

\subsection{Data Collection}

We collected a set of human-human dialogues using Amazon Mechanical Turk. Workers were paid $\$ 0.15$ per dialogue, with a $\$ 0.05$ bonus for maximal scores. We only used workers based in the United States with a 95\% approval rating and at least 5000 previous HITs. Our data collection interface was adapted from that of Das et al. (2016). 

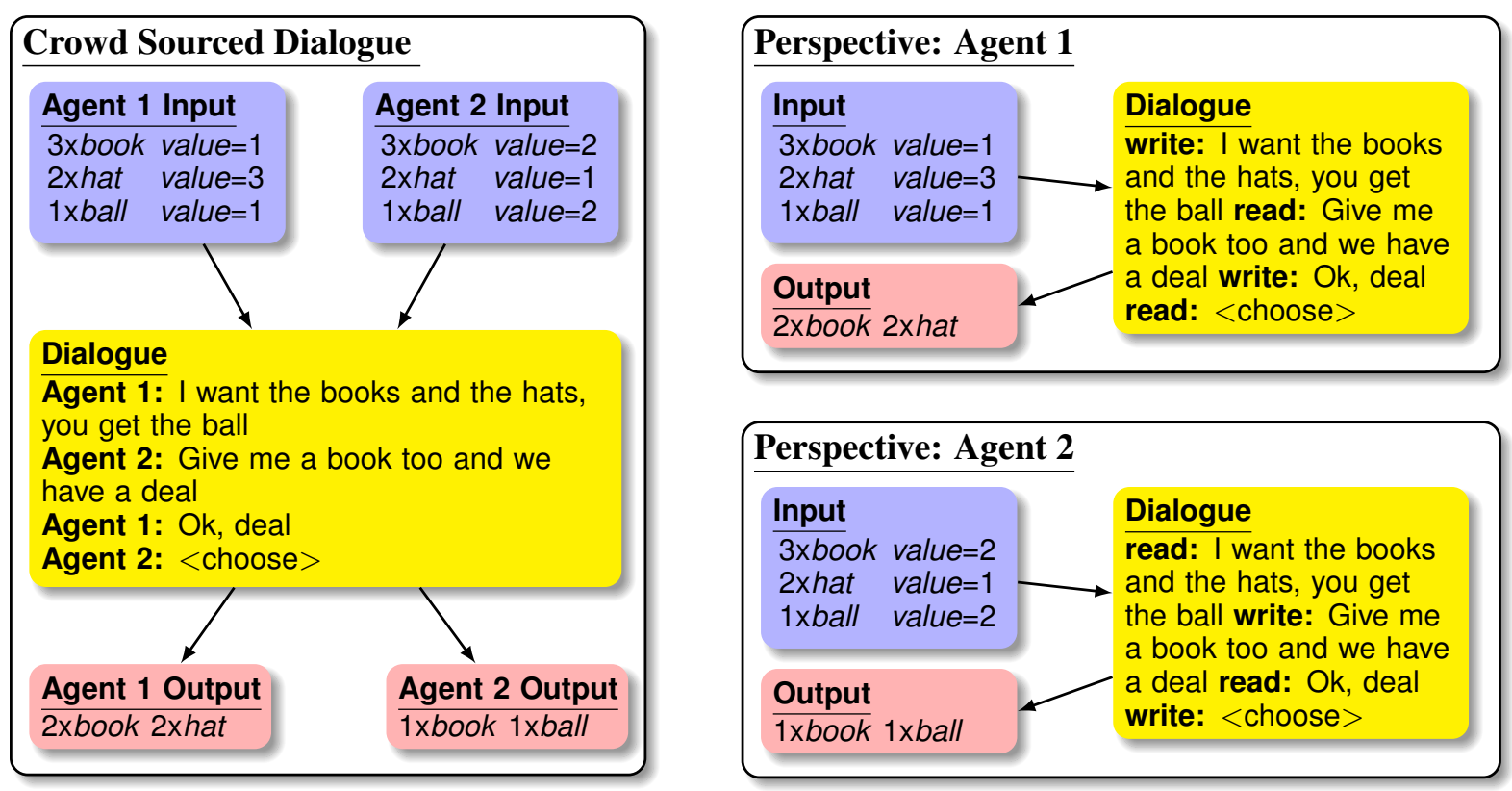

Figure 2: Converting a crowd-sourced dialogue (left) into two training examples (right), from the perspective of each user. The perspectives differ on their input goals, output choice, and in special tokens marking whether a statement was read or written. We train conditional language models to predict the dialogue given the input, and additional models to predict the output given the dialogue.

We collected a total of 5808 dialogues, based on 2236 unique scenarios (where a scenario is the available items and values for the two users). We held out a test set of 252 scenarios ( 526 dialogues). Holding out test scenarios means that models must generalise to new situations.

\section{Likelihood Model}

We propose a simple but effective baseline model for the conversational agent, in which a sequenceto-sequence model is trained to produce the complete dialogue, conditioned on an agent's input.

\subsection{Data Representation}

Each dialogue is converted into two training examples, showing the complete conversation from the perspective of each agent. The examples differ on their input goals, output choice, and whether utterances were read or written.

Training examples contain an input goal $g$, specifying the available items and their values, a dialogue $x$, and an output decision $o$ specifying which items each agent will receive. Specifically, we represent $g$ as a list of six integers corresponding to the count and value of each of the three item types. Dialogue $x$ is a list of tokens $x_{0 . . T}$ containing the turns of each agent interleaved with symbols marking whether a turn was written by the agent or their partner, terminating in a special token indicating one agent has marked that an agreement has been made. Output $o$ is six integers describing how many of each of the three item types are assigned to each agent. See Figure 2.

\subsection{Supervised Learning}

We train a sequence-to-sequence network to generate an agent's perspective of the dialogue conditioned on the agent's input goals (Figure 3a).

The model uses 4 recurrent neural networks, implemented as GRUs (Cho et al., 2014): $\mathrm{GRU}_{w}$, $\mathrm{GRU}_{g}, \mathrm{GRU}_{\vec{o}}$, and $\mathrm{GRU}_{\overleftarrow{o}}$.

The agent's input goals $g$ are encoded using $\mathrm{GRU}_{g}$. We refer to the final hidden state as $h^{g}$. The model then predicts each token $x_{t}$ from left to right, conditioned on the previous tokens and $h^{g}$. At each time step $t, \mathrm{GRU}_{w}$ takes as input the previous hidden state $h_{t-1}$, previous token $x_{t-1}$ (embedded with a matrix $E$ ), and input encoding $h^{g}$. Conditioning on the input at each time step helps the model learn dependencies between language and goals.

$$
h_{t}=\mathrm{GRU}_{w}\left(h_{t-1},\left[E x_{t-1}, h^{g}\right]\right)
$$

The token at each time step is predicted with a softmax, which uses weight tying with the embed- 


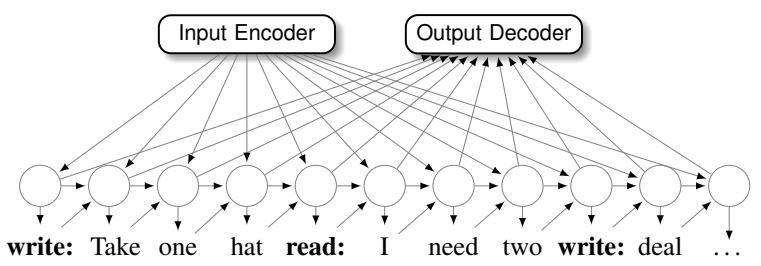

(a) Supervised Training

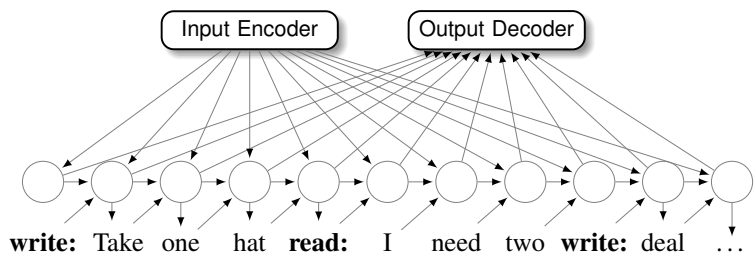

(b) Decoding, and Reinforcement Learning

Figure 3: Our model: tokens are predicted conditioned on previous words and the input, then the output is predicted using attention over the complete dialogue. In supervised training (3a), we train the model to predict the tokens of both agents. During decoding and reinforcement learning (3b) some tokens are sampled from the model, but some are generated by the other agent and are only encoded by the model.

ding matrix $E$ (Mao et al., 2015):

$$
p_{\theta}\left(x_{t} \mid x_{0 . . t-1}, g\right) \propto \exp \left(E^{T} h_{t}\right)
$$

Note that the model predicts both agent's words, enabling its use as a forward model in Section 5.

At the end of the dialogue, the agent outputs a set of tokens $o$ representing the decision. We generate each output conditionally independently, using a separate classifier for each. The classifiers share bidirectional GRUs and an attention mechanism (Bahdanau et al., 2014) over the dialogue, and additionally condition on the input goals.

$$
\begin{aligned}
h_{t}^{\vec{o}} & =\operatorname{GRU}_{\vec{o}}\left(h_{t-1}^{\vec{o}},\left[E x_{t}, h_{t}\right]\right) \\
h_{t}^{\overleftarrow{o}} & =\operatorname{GRU}_{\overleftarrow{o}}\left(h_{t+1}^{\overleftarrow{o}},\left[E x_{t}, h_{t}\right]\right) \\
h_{t}^{o} & =\left[h_{t}^{\overleftarrow{o}}, h_{t}^{\vec{o}}\right] \\
h_{t}^{a} & =W^{a}\left[\tanh \left(W^{h} h_{t}^{o}\right)\right] \\
\alpha_{t} & =\frac{\exp \left(w \cdot h_{t}^{a}\right)}{\sum_{t^{\prime}} \exp \left(w \cdot h_{t^{\prime}}^{a}\right)} \\
h^{s} & =\tanh \left(W^{s}\left[h^{g}, \sum_{t} \alpha_{t} h_{t}\right]\right)
\end{aligned}
$$

The output tokens are predicted using softmax:

$$
p_{\theta}\left(o_{i} \mid x_{0 . t}, g\right) \propto \exp \left(W^{o_{i}} h^{s}\right)
$$

The model is trained to minimize the negative $\log$ likelihood of the token sequence $x_{0 . . T}$ conditioned on the input goals $g$, and of the outputs $o$ conditioned on $x$ and $g$. The two terms are weighted with a hyperparameter $\alpha$.

$$
\begin{aligned}
L(\theta)= & -\underbrace{\sum_{x, g} \sum_{t} \log p_{\theta}\left(x_{t} \mid x_{0 . . t-1}, g\right)}_{\text {Token prediction loss }} \\
& -\alpha \underbrace{\alpha \sum_{x, g, o} \sum_{j} \log p_{\theta}\left(o_{j} \mid x_{0 . . T}, g\right)}_{\text {Output choice prediction loss }}
\end{aligned}
$$

Unlike the Neural Conversational Model (Vinyals and Le, 2015), our approach shares all parameters for reading and generating tokens.

\subsection{Decoding}

During decoding, the model must generate an output token $x_{t}$ conditioned on dialogue history $x_{0 . t-1}$ and input goals $g$, by sampling from $p_{\theta}$ :

$$
x_{t} \sim p_{\theta}\left(x_{t} \mid x_{0 . . t-1}, g\right)
$$

If the model generates a special end-of-turn token, it then encodes a series of tokens output by the other agent, until its next turn (Figure 3b).

The dialogue ends when either agent outputs a special end-of-dialogue token. The model then outputs a set of choices $o$. We choose each item independently, but enforce consistency by checking the solution is in a feasible set $O$ :

$$
o^{*}=\underset{o \in O}{\operatorname{argmax}} \prod_{i} p_{\theta}\left(o_{i} \mid x_{0 . . T}, g\right)
$$

In our task, a solution is feasible if each item is assigned to exactly one agent. The space of solutions is small enough to be tractably enumerated.

\section{Goal-based Training}

Supervised learning aims to imitate the actions of human users, but does not explicitly attempt to maximise an agent's goals. Instead, we explore pre-training with supervised learning, and then fine-tuning against the evaluation metric using reinforcement learning. Similar two-stage learning strategies have been used previously (e.g. Li et al. (2016); Das et al. (2017)).

During reinforcement learning, an agent $A$ attempts to improve its parameters from conversations with another agent $B$. While the other agent $B$ could be a human, in our experiments we used 


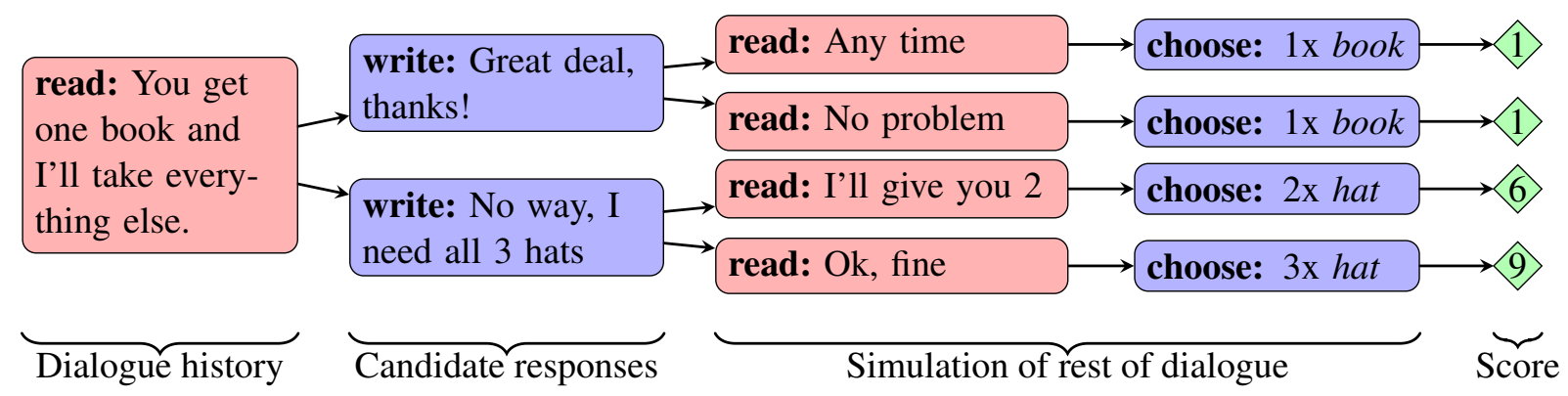

Figure 4: Decoding through rollouts: The model first generates a small set of candidate responses. For each candidate, it then simulates a number of possible complete future conversations by sampling, and estimates the expected future reward by averaging the scores. The system outputs the candidate with the highest expected reward.

our fixed supervised model that was trained to imitate humans. The second model is fixed as we found that updating the parameters of both agents led to divergence from human language. In effect, agent $A$ learns to improve by simulating conversations with the help of a surrogate forward model.

Agent $A$ reads its goals $g$ and then generates tokens $x_{0 . . n}$ by sampling from $p_{\theta}$. When $x$ generates an end-of-turn marker, it then reads in tokens $x_{n+1 . . m}$ generated by agent $B$. These turns alternate until one agent emits a token ending the dialogue. Both agents then output a decision $o$ and collect a reward from the environment (which will be 0 if they output different decisions). We denote the subset of tokens generated by $A$ as $X^{A}$ (e.g. tokens with incoming arrows in Figure $3 b$ ).

After a complete dialogue has been generated, we update agent $A$ 's parameters based on the outcome of the negotiation. Let $r^{A}$ be the score agent $A$ achieved in the completed dialogue, $T$ be the length of the dialogue, $\gamma$ be a discount factor that rewards actions at the end of the dialogue more strongly, and $\mu$ be a running average of completed dialogue rewards so $\mathrm{far}^{2}$. We define the future reward $R$ for an action $x_{t} \in X^{A}$ as follows:

$$
R\left(x_{t}\right)=\sum_{x_{t} \in X^{A}} \gamma^{T-t}\left(r^{A}(o)-\mu\right)
$$

We then optimise the expected reward of each action $x_{t} \in X^{A}$ :

$$
L_{\theta}^{R L}=\mathbb{E}_{x_{t} \sim p_{\theta}\left(x_{t} \mid x_{0 . . t-1}, g\right)}\left[R\left(x_{t}\right)\right]
$$

\footnotetext{
${ }^{2}$ As all rewards are non-negative, we instead re-scale them by subtracting the mean reward found during self play. Shifting in this way can reduce the variance of our estimator.
}

The gradient of $L_{\theta}^{R L}$ is calculated as in REINFORCE (Williams, 1992):

$$
\nabla_{\theta} L_{\theta}^{R L}=\sum_{x_{t} \in X^{A}} \mathbb{E}_{x_{t}}\left[R\left(x_{t}\right) \nabla_{\theta} \log \left(p_{\theta}\left(x_{t} \mid x_{0 . . t-1}, g\right)\right)\right]
$$

\section{Goal-based Decoding}

Likelihood-based decoding (§3.3) may not be optimal. For instance, an agent may be choosing between accepting an offer, or making a counter offer. The former will often have a higher likelihood under our model, as there are fewer ways to agree than to make another offer, but the latter may lead to a better outcome. Goal-based decoding also allows more complex dialogue strategies. For example, a deceptive utterance is likely to have a low model score (as users were generally honest in the supervised data), but may achieve high reward.

We instead explore decoding by maximising expected reward. We achieve this by using $p_{\theta}$ as a forward model for the complete dialogue, and then deterministically computing the reward. Rewards for an utterance are averaged over samples to calculate expected future reward (Figure 4).

We use a two stage process: First, we generate $c$ candidate utterances $U=u_{0 . . c}$, representing possible complete turns that the agent could make, which are generated by sampling from $p_{\theta}$ until the end-of-turn token is reached. Let $x_{0 . . n-1}$ be current dialogue history. We then calculate the expected reward $R(u)$ of candidate utterance $u=x_{n, n+k}$ by repeatedly sampling $x_{n+k+1, T}$ from $p_{\theta}$, then choosing the best output $o$ using Equation 12, and finally deterministically computing the reward $r(o)$. The reward is scaled by the probability of the output given the dialogue, be- 


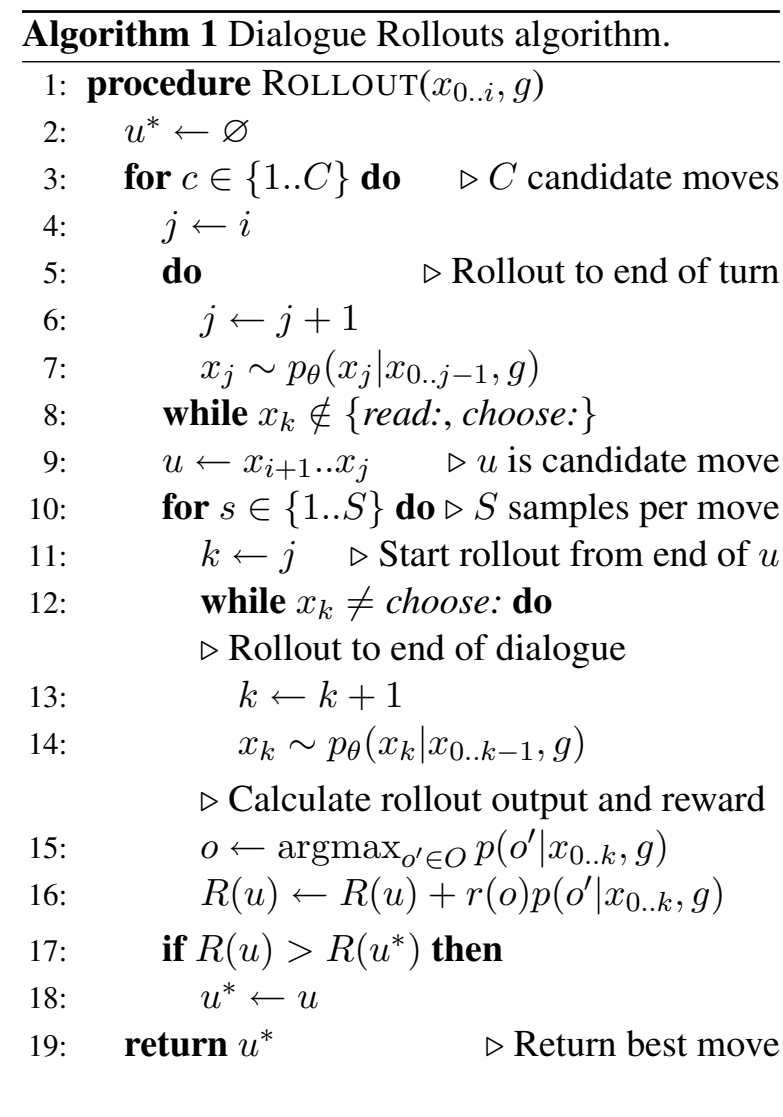

cause if the agents select different outputs then they both receive 0 reward.

$$
R\left(x_{n . . n+k}\right)=\mathbb{E}_{x_{(n+k+1 . . T ; o)} \sim p_{\theta}}\left[r(o) p_{\theta}\left(o \mid x_{0 . . T}\right)\right]
$$

We then return the utterance maximizing $R$.

$$
u^{*}=\underset{u \in U}{\operatorname{argmax}} R(u)
$$

We use 5 rollouts for each of 10 candidate turns.

\section{Experiments}

\subsection{Training Details}

We implement our models using PyTorch. All hyper-parameters were chosen on a development dataset. The input tokens are embedded into a 64-dimensional space, while the dialogue tokens are embedded with 256-dimensional embeddings (with no pre-training). The input $G R U_{g}$ has a hidden layer of size 64 and the dialogue $G R U_{w}$ is of size 128. The output GRU $\vec{o}_{o}$ and GRU $\overleftarrow{\leftarrow}$ both have a hidden state of size 256 , the size of $h^{s}$ is 256 as well. During supervised training, we optimise using stochastic gradient descent with a minibatch size of 16 , an initial learning rate of 1.0 , Nesterov momentum with $\mu=0.1$ (Nesterov,
1983), and clipping gradients whose $L^{2}$ norm exceeds 0.5 . We train the model for 30 epochs and pick the snapshot of the model with the best validation perplexity. We then annealed the learning rate by a factor of 5 each epoch. We weight the terms in the loss function (Equation 10) using $\alpha=0.5$. We do not train against output decisions where humans selected different agreements. Tokens occurring fewer than 20 times are replaced with an 'unknown' token.

During reinforcement learning, we use a learning rate of 0.1 , clip gradients above 1.0, and use a discount factor of $\gamma=0.95$. After every 4 reinforcement learning updates, we make a supervised update with mini-batch size 16 and learning rate 0.5 , and we clip gradients at 1.0. We used 4086 simulated conversations.

When sampling words from $p_{\theta}$, we reduce the variance by doubling the values of logits (i.e. using temperature of 0.5 ).

\subsection{Comparison Systems}

We compare the performance of the following: LIKELIHOOD uses supervised training and decoding (§3), RL is fine-tuned with goal-based selfplay ( $(4)$, ROLLOUTS uses supervised training combined with goal-based decoding using rollouts ( $(5)$, and RL+ROLLOUTS uses rollouts with a base model trained with reinforcement learning.

\subsection{Intrinsic Evaluation}

For development, we use measured the perplexity of user generated utterances, conditioned on the input and previous dialogue.

Results are shown in Table 3, and show that the simple LIKELIHOOD model produces the most human-like responses, and the alternative training and decoding strategies cause a divergence from human language. Note however, that this divergence may not necessarily correspond to lower quality language - it may also indicate different strategic decisions about what to say. Results in $\S 6.4$ show all models could converse with humans.

\subsection{End-to-End Evaluation}

We measure end-to-end performance in dialogues both with the likelihood-based agent and with humans on Mechanical Turk, on held out scenarios.

Humans were told that they were interacting with other humans, as they had been during the collection of our dataset (and few appeared to realize they were in conversation with machines). 


\begin{tabular}{|c|cccc|cccc|}
\cline { 2 - 9 } \multicolumn{1}{c|}{} & \multicolumn{4}{c|}{ vs. LIKELIHOOD } & \multicolumn{4}{c|}{ vs. Human } \\
\hline \multirow{2}{*}{ Model } & $\begin{array}{c}\text { Score } \\
\text { (all) }\end{array}$ & $\begin{array}{c}\text { Score } \\
\text { (agreed) }\end{array}$ & $\begin{array}{c}\% \\
\text { Agreed }\end{array}$ & $\begin{array}{c}\text { \% Pareto } \\
\text { Optimal }\end{array}$ & $\begin{array}{c}\text { Score } \\
\text { (all) }\end{array}$ & $\begin{array}{c}\text { Score } \\
\text { (agreed) }\end{array}$ & $\begin{array}{c}\% \\
\text { Agreed }\end{array}$ & $\begin{array}{c}\text { \% Pareto } \\
\text { Optimal }\end{array}$ \\
\hline LIKELIHOOD & 5.4 vs. 5.5 & 6.2 vs. 6.2 & 87.9 & 49.6 & 4.7 vs. 5.8 & 6.2 vs. 7.6 & $\mathbf{7 6 . 5}$ & 66.2 \\
RL & 7.1 vs. 4.2 & 7.9 vs. 4.7 & 89.9 & 58.6 & 4.3 vs. 5.0 & 6.4 vs. 7.5 & 67.3 & 69.1 \\
ROLLOUTS & 7.3 vs. 5.1 & 7.9 vs. 5.5 & 92.9 & 63.7 & $\mathbf{5 . 2}$ vs. 5.4 & 7.1 vs. 7.4 & 72.1 & 78.3 \\
RL+ROLLOUTS & $\mathbf{8 . 3}$ vs. $\mathbf{4 . 2}$ & $\mathbf{8 . 8}$ vs. $\mathbf{4 . 5}$ & $\mathbf{9 4 . 4}$ & $\mathbf{7 4 . 8}$ & 4.6 vs. 4.2 & $\mathbf{8 . 0}$ vs. 7.1 & 57.2 & $\mathbf{8 2 . 4}$ \\
\hline
\end{tabular}

Table 1: End task evaluation on heldout scenarios, against the LIKELIHOOD model and humans from Mechanical Turk. The maximum score is 10 . Score (all) gives 0 points when agents failed to agree.

\begin{tabular}{|c|c|}
\hline Metric & Dataset \\
\hline Number of Dialogues & 5808 \\
Average Turns per Dialogue & 6.6 \\
Average Words per Turn & 7.6 \\
\% Agreed & 80.1 \\
Average Score (/10) & 6.0 \\
\% Pareto Optimal & 76.9 \\
\hline
\end{tabular}

Table 2: Statistics on our dataset of crowdsourced dialogues between humans.

\begin{tabular}{|c|c|c|c|}
\hline Model & Valid PPL & Test PPL & Test Avg. Rank \\
\hline LIKELIHOOD & 5.62 & 5.47 & 521.8 \\
RL & 6.03 & 5.86 & 517.6 \\
ROLLOUTS & - & - & 844.1 \\
RL+ROLLOUTS & - & - & 859.8 \\
\hline
\end{tabular}

Table 3: Intrinsic evaluation showing the average perplexity of tokens and rank of complete turns (out of 2083 unique human messages from the test set). Lower is more human-like for both.

We measure the following statistics:

Score: The average score for each agent (which could be a human or model), out of 10 .

Agreement: The percentage of dialogues where both agents agreed on the same decision.

Pareto Optimality: The percentage of Pareto optimal solutions for agreed deals (a solution is Pareto optimal if neither agent's score can be improved without lowering the other's score). Lower scores indicate inefficient negotiations.

Results are shown in Table 1. Firstly, we see that the RL and ROLLOUTS models achieve significantly better results when negotiating with the LIKELIHOOD model, particularly the RL+ROLLOUTS model. The percentage of Pareto optimal solutions also increases, showing a better exploration of the solution space. Compared to human-human negotiations (Table 2), the best models achieve a higher agreement rate, better scores, and similar Pareto efficiency. This result confirms that attempting to maximise reward can outperform simply imitating humans.

Similar trends hold in dialogues with humans, with goal-based reasoning outperforming imitation learning. The ROLLOUTS model achieves comparable scores to its human partners, and the RL+ROLLOUTS model actually achieves higher scores. However, we also find significantly more cases of the goal-based models failing to agree a deal with humans-largely a consequence of their more aggressive negotiation tactics (see $\S 7$ ).

\section{Analysis}

Table 1 shows large gains from goal-based methods. In this section, we explore the strengths and weaknesses of our models.

Goal-based models negotiate harder. The RL+ROLLOUTS model has much longer dialogues with humans than LIKELIHOOD (7.2 turns vs. 5.3 on average), indicating that the model is accepting deals less quickly, and negotiating harder.

A negative consequence of more aggressive negotiation strategies is that humans were more likely to walk away with no deal, resulting in lower agreement rates. Even though failing to agree was worth 0 points, people often preferred this course over capitulating to an uncompromising opponent-a factor not well captured by the simulated partner in reinforcement learning training or rollouts (as reflected by the larger gains from goal-based models in dialogues with the LIKELIHOOD model). In particular, the goal-based models are prone to simply rephrasing the same demand each turn, which is a more effective strategy against the LIKELIHOOD model than humans.

One approach to mitigating this issue would be to learn the impact of uncooperative strategies across multiple rounds of negotiation (Lerer and Peysakhovich, 2017). In single dialogues, failing to agree is never better than reaching some agreement. However, across multiple rounds, learning to walk away without a deal may incentivize partners to cooperate more in future.

Figure 5 shows an example of our goal-based 


\begin{tabular}{|c|c|}
\hline \multicolumn{2}{|l|}{ Input } \\
\hline Human & 1 xbook value $=31$ xhat value $=13$ xball value $=2$ \\
\hline RL+ROLLOUTS & $1 \mathrm{xbook}$ value $=61 \mathrm{xhat}$ value $=43 \mathrm{xball}$ value $=0$ \\
\hline RL+ROLLOUTS & You can have the balls if $i$ can have the hat and book \\
\hline Human & $\begin{array}{l}\text { Nope, give me the books and you can have } \\
\text { everything else }\end{array}$ \\
\hline RL+ROLLOUTS & Ok. I will take the book and one hat \\
\hline Human & I get all the balls? \\
\hline RL+ROLLOUTS & Yes. I can do that. \\
\hline Human & Ok, deal \\
\hline Output & Reward \\
\hline RL+ROLLOUTS & 1xbook 1xhat \\
\hline Human & $3 x$ ball \\
\hline
\end{tabular}

Figure 5: Example of a model stubbornly holding out for maximum points in a dialogue.

\begin{tabular}{|c|c|c|}
\hline Input & & \\
\hline Human & \multirow{2}{*}{\multicolumn{2}{|c|}{$\begin{array}{l}3 \text { xbook } \text { value }=2 \text { 3xhat } \text { value }=11 \text { xball value }=1 \\
3 \text { xbook } \text { value }=13 \text { xhat } \text { value }=21 \text { xball } \text { value }=1\end{array}$}} \\
\hline RL+ROLLOUTS & & \\
\hline Human & \multicolumn{2}{|c|}{ I'd like the books and the hats. } \\
\hline RL+ROLLOUTS & \multicolumn{2}{|c|}{ I will take the books and hats } \\
\hline Human & \multirow{4}{*}{\multicolumn{2}{|c|}{$\begin{array}{l}\text { I really need the books and at least one other object. } \\
\text { Ok, you can have one book and one ball } \\
\text { If I can have all the books, I can leave you the rest. } \\
\text { Deal }\end{array}$}} \\
\hline RL+ROLLOUTS & & \\
\hline Human & & \\
\hline RL+ROLLOUTS & & \\
\hline Output & & Reward \\
\hline RL+ROLLOUTS & $3 x$ hat 1 xball & $7 / 10$ \\
\hline Human & 3 xbook & $6 / 10$ \\
\hline
\end{tabular}

Figure 6: Example of model compromising.

model stubbornly negotiating until it achieves a good outcome.

Models learn to be deceptive. Deception can be an effective negotiation tactic. We found numerous cases of our models initially feigning interest in a valueless item, only to later 'compromise' by conceding it. Figure 7 shows an example. This strategy has previously been coded into negotiation agents by Gratch et al. (2016), whereas our agents have learned it from data.

Models produce meaningful novel sentences. One interesting question is whether our models are capable of generating novel sentences in the new circumstances they find themselves in, or if they simply repeat messages from the training data verbatim. We find that $76 \%$ of messages produced by the LIKELIHOOD model in self-play were found in the training data. We manually examined the novel utterances produced by our model, and found that the overwhelming majority were fluent English sentences in isolation-showing that the model has learnt a good language model for the domain (in addition to results that show it uses language effectively to achieve its goals). These results suggest that although neural models are prone to the safer option of repeating sentences from training data, they are capable of generalising when nec-

\begin{tabular}{|c|c|}
\hline Input & \\
\hline RL+ROLLOUTS & 4 xbook value $=02$ hat value $=51$ xball value $=0$ \\
\hline Human & 4 xbook value $=12$ hat value $=21$ xball value $=2$ \\
\hline RL+ROLLOUTS & You can have the ball if I can have everything else. \\
\hline Human & $\begin{array}{l}\text { No can't do that the ball is not going to give } \\
\text { me anything }\end{array}$ \\
\hline RL+ROLLOUTS & I need the hats and you can have the rest \\
\hline Human & Deal \\
\hline RL+ROLLOUTS & Great! Thank you! \\
\hline Output & Reward \\
\hline RL+ROLLOUTS & 2xhat \\
\hline Human & 4 xbook 1 xball \\
\hline
\end{tabular}

Figure 7: Dialogue in which the model's initial interest in the valueless books allows it to compromise while achieving a maximum score.

essary. Future work should choose domains that force a higher degree of diversity in utterances.

Maintaining multi-sentence coherence is challenging. One common linguistic error we see RL+ROLLOUTS make is to start a message by indicating agreement (e.g. I agree or Deal), but then going on to propose a counter offer-a behaviour that human partners found frustrating. One explanation is that the model has learnt that in the supervised data, messages beginning with I agree are often at the end of the dialogue, and partners rarely reply with further negotiation-so the models using rollouts and reinforcement learning believe this tactic will help their offer to be accepted.

\section{Related Work}

Most work on goal orientated dialogue systems has assumed that state representations are annotated in the training data (Williams and Young, 2007; Henderson et al., 2014; Wen et al., 2016). The use of state annotations allows a cleaner separation of the reasoning and natural language aspects of dialogues, but our end-to-end approach makes data collection cheaper and allows tasks where it is unclear how to annotate state. Bordes and Weston (2016) explore end-to-end goal orientated dialogue with a supervised model-we show improvements over supervised learning with goalbased training and decoding. Recently, He et al. (2017) use task-specific rules to combine the task input and dialogue history into a more structured state representation than ours.

Reinforcement learning (RL) has been applied in many dialogue settings. RL has been widely used to improve dialogue managers, which manage transitions between dialogue states (Singh et al., 2002; Pietquin et al., 2011; Rieser and Lemon, 2011; Gašic et al., 2013; Fatemi et al., 
2016). In contrast, our end-to-end approach has no explicit dialogue manager that can be updated in isolation, and we found it necessary to interleave RL and supervised learning to avoid RL reducing the quality of language generation. Li et al. (2016) improve metrics such as diversity for nongoal-orientated dialogue using RL, which would make an interesting extension to our work. Das et al. (2017) use reinforcement learning to improve cooperative bot-bot dialogues. RL has also been used to allow agents to invent new languages (Das et al., 2017; Mordatch and Abbeel, 2017). To our knowledge, our model is the first to use RL to improve the performance of an end-to-end goal orientated dialogue system in dialogues with humans.

Work on learning end-to-end dialogues has concentrated on 'chat' settings, without explicit goals (Ritter et al., 2011; Vinyals and Le, 2015; Li et al., 2015). These dialogues contain a much greater diversity of vocabulary than our domain, but do not have the challenging adversarial elements. Such models are notoriously hard to evaluate (Liu et al., 2016), because the huge diversity of reasonable responses, whereas our task has a clear objective. Our end-to-end approach would also be much more straightforward to integrate into a generalpurpose dialogue agent than one that relied on annotated dialogue states (Dodge et al., 2016).

There is a substantial literature on multi-agent bargaining in game-theory, e.g. Nash Jr (1950). There has also been computational work on modelling negotiations (Baarslag et al., 2013)—our work differs in that agents communicate in unrestricted natural language, rather than pre-specified symbolic actions, and our focus on improving performance relative to humans rather than other automated systems. Our task is based on that of DeVault et al. (2015), who study natural language negotiations for pedagogical purposes- their version includes speech rather than textual dialogue, and embodied agents, which would make interesting extensions to our work. The only automated natural language negotiations systems we are aware of have first mapped language to domain-specific logical forms, and then focused on choosing the next dialogue act (Rosenfeld et al., 2014; Cuayáhuitl et al., 2015; Keizer et al., 2017). Our end-to-end approach is the first to learn comprehension, reasoning and generation skills in a domain-independent data driven way.

Our use of a combination of supervised and re- inforcement learning for training, and stochastic rollouts for decoding, builds on strategies used in game playing agents such as AlphaGo (Silver et al., 2016). Our work is a step towards realworld applications for these techniques. Our use of rollouts could be extended by choosing the other agent's responses based on sampling, using Monte Carlo Tree Search (MCTS) (Kocsis and Szepesvári, 2006). However, our setting has a higher branching factor than in domains where MCTS has been successfully applied, such as Go (Silver et al., 2016)—future work should explore scaling tree search to dialogue modelling.

\section{Conclusion}

We have introduced end-to-end learning of natural language negotiations as a task for AI, arguing that it challenges both linguistic and reasoning skills while having robust evaluation metrics. We gathered a large dataset of human-human negotiations, which contain a variety of interesting tactics. We have shown that it is possible to train dialogue agents end-to-end, but that their ability can be much improved by training and decoding to maximise their goals, rather than likelihood. There remains much potential for future work, particularly in exploring other reasoning strategies, and in improving the diversity of utterances without diverging from human language. We will also explore other negotiation tasks, to investigate whether models can learn to share negotiation strategies across domains.

\section{Acknowledgments}

We would like to thank Luke Zettlemoyer and the anonymous EMNLP reviewers for their insightful comments, and the Mechanical Turk workers who helped us collect data.

\section{References}

Nicholas Asher, Alex Lascarides, Oliver Lemon, Markus Guhe, Verena Rieser, Philippe Muller, Stergos Afantenos, Farah Benamara, Laure Vieu, Pascal Denis, et al. 2012. Modelling Strategic Conversation: The STAC project. Proceedings of SemDial, page 27.

Tim Baarslag, Katsuhide Fujita, Enrico H Gerding, Koen Hindriks, Takayuki Ito, Nicholas R Jennings, Catholijn Jonker, Sarit Kraus, Raz Lin, Valentin Robu, et al. 2013. Evaluating Practical Negotiating 
Agents: Results and Analysis of the 2011 International Competition. Artificial Intelligence, 198:73103.

Dzmitry Bahdanau, Kyunghyun Cho, and Yoshua Bengio. 2014. Neural Machine Translation by Jointly Learning to Align and Translate. arXiv preprint arXiv:1409.0473.

Antoine Bordes and Jason Weston. 2016. Learning End-to-End Goal-oriented Dialog. arXiv preprint arXiv:1605.07683.

Kyunghyun Cho, Bart Van Merriënboer, Dzmitry Bahdanau, and Yoshua Bengio. 2014. On the Properties of Neural Machine Translation: Encoder-decoder Approaches. arXiv preprint arXiv:1409.1259.

Heriberto Cuayáhuitl, Simon Keizer, and Oliver Lemon. 2015. Strategic Dialogue Management via Deep Reinforcement Learning. arXiv preprint arXiv:1511.08099.

Abhishek Das, Satwik Kottur, Khushi Gupta, Avi Singh, Deshraj Yadav, José MF Moura, Devi Parikh, and Dhruv Batra. 2016. Visual Dialog. arXiv preprint arXiv:1611.08669.

Abhishek Das, Satwik Kottur, José MF Moura, Stefan Lee, and Dhruv Batra. 2017. Learning Cooperative Visual Dialog Agents with Deep Reinforcement Learning. arXiv preprint arXiv:1703.06585.

David DeVault, Johnathan Mell, and Jonathan Gratch. 2015. Toward Natural Turn-taking in a Virtual $\mathrm{Hu}-$ man Negotiation Agent. In AAAI Spring Symposium on Turn-taking and Coordination in HumanMachine Interaction. AAAI Press, Stanford, CA.

Jesse Dodge, Andreea Gane, Xiang Zhang, Antoine Bordes, Sumit Chopra, Alexander H. Miller, Arthur Szlam, and Jason Weston. 2016. Evaluating Prerequisite Qualities for Learning End-to-End Dialog Systems. ICLR, abs/1511.06931.

Mehdi Fatemi, Layla El Asri, Hannes Schulz, Jing He, and Kaheer Suleman. 2016. Policy Networks with Two-stage Training for Dialogue Systems. arXiv preprint arXiv:1606.03152.

Chaim Fershtman. 1990. The Importance of the Agenda in Bargaining. Games and Economic Behavior, 2(3):224-238.

Milica Gašic, Catherine Breslin, Matthew Henderson, Dongho Kim, Martin Szummer, Blaise Thomson, Pirros Tsiakoulis, and Steve Young. 2013. POMDPbased Dialogue Manager Adaptation to Extended Domains. In Proceedings of SIGDIAL.

Jonathan Gratch, Zahra Nazari, and Emmanuel Johnson. 2016. The Misrepresentation Game: How to win at negotiation while seeming like a nice guy. In Proceedings of the 2016 International Conference on Autonomous Agents \& Multiagent Systems, pages 728-737. International Foundation for Autonomous Agents and Multiagent Systems.
H. He, A. Balakrishnan, M. Eric, and P. Liang. 2017. Learning Symmetric Collaborative Dialogue Agents with Dynamic Knowledge Graph Embeddings. In Association for Computational Linguistics (ACL).

Matthew Henderson, Blaise Thomson, and Jason Williams. 2014. The Second Dialog State Tracking Challenge. In 15th Annual Meeting of the Special Interest Group on Discourse and Dialogue, volume 263.

Simon Keizer, Markus Guhe, Heriberto Cuayáhuitl, Ioannis Efstathiou, Klaus-Peter Engelbrecht, Mihai Dobre, Alexandra Lascarides, and Oliver Lemon. 2017. Evaluating Persuasion Strategies and Deep Reinforcement Learning methods for Negotiation Dialogue agents. In Proceedings of the European Chapter of the Association for Computational Linguistics (EACL 2017).

Levente Kocsis and Csaba Szepesvári. 2006. Bandit based Monte-Carlo Planning. In European conference on machine learning, pages 282-293. Springer.

Adam Lerer and Alexander Peysakhovich. 2017. Maintaining Cooperation in Complex Social Dilemmas using Deep Reinforcement Learning. arXiv preprint arXiv:1707.01068.

Jiwei Li, Michel Galley, Chris Brockett, Jianfeng Gao, and Bill Dolan. 2015. A Diversity-promoting Objective Function for Neural Conversation Models. arXiv preprint arXiv:1510.03055.

Jiwei Li, Will Monroe, Alan Ritter, Michel Galley, Jianfeng Gao, and Dan Jurafsky. 2016. Deep Reinforcement Learning for Dialogue Generation. arXiv preprint arXiv:1606.01541.

Chia-Wei Liu, Ryan Lowe, Iulian V. Serban, Michael Noseworthy, Laurent Charlin, and Joelle Pineau. 2016. How NOT To Evaluate Your Dialogue System: An Empirical Study of Unsupervised Evaluation Metrics for Dialogue Response Generation. In Proceedings of the Conference on Empirical Methods in Natural Language Processing.

Junhua Mao, Xu Wei, Yi Yang, Jiang Wang, Zhiheng Huang, and Alan L. Yuille. 2015. Learning Like a Child: Fast Novel Visual Concept Learning From Sentence Descriptions of Images. In The IEEE International Conference on Computer Vision (ICCV).

Igor Mordatch and Pieter Abbeel. 2017. Emergence of Grounded Compositional Language in Multi-Agent Populations. arXiv preprint arXiv:1703.04908.

John F Nash Jr. 1950. The Bargaining Problem. Econometrica: Journal of the Econometric Society, pages 155-162.

Yurii Nesterov. 1983. A Method of Solving a Convex Programming Problem with Convergence Rate O (1/k2). In Soviet Mathematics Doklady, volume 27, pages $372-376$. 
Olivier Pietquin, Matthieu Geist, Senthilkumar Chandramohan, and Hervé Frezza-Buet. 2011. Sampleefficient Batch Reinforcement Learning for Dialogue Management Optimization. ACM Trans. Speech Lang. Process., 7(3):7:1-7:21.

Verena Rieser and Oliver Lemon. 2011. Reinforcement Learning for Adaptive Dialogue Systems: A Datadriven Methodology for Dialogue Management and Natural Language Generation. Springer Science \& Business Media.

Alan Ritter, Colin Cherry, and William B Dolan. 2011. Data-driven Response Generation in Social Media. In Proceedings of the Conference on Empirical Methods in Natural Language Processing, pages 583-593. Association for Computational Linguistics.

Avi Rosenfeld, Inon Zuckerman, Erel Segal-Halevi, Osnat Drein, and Sarit Kraus. 2014. NegoChat: A Chat-based Negotiation Agent. In Proceedings of the 2014 International Conference on Autonomous Agents and Multi-agent Systems, AAMAS '14, pages 525-532, Richland, SC. International Foundation for Autonomous Agents and Multiagent Systems.

David Silver, Aja Huang, Chris J Maddison, Arthur Guez, Laurent Sifre, George Van Den Driessche, Julian Schrittwieser, Ioannis Antonoglou, Veda Panneershelvam, Marc Lanctot, et al. 2016. Mastering the Game of Go with Deep Neural Networks and Tree Search. Nature, 529(7587):484-489.

Satinder Singh, Diane Litman, Michael Kearns, and Marilyn Walker. 2002. Optimizing Dialogue Management with Reinforcement Learning: Experiments with the NJFun System. Journal of Artificial Intelligence Research, 16:105-133.

Victoria Talwar and Kang Lee. 2002. Development of lying to conceal a transgression: Children's control of expressive behaviour during verbal deception. International Journal of Behavioral Development, 26(5):436-444.

David Traum, Stacy C. Marsella, Jonathan Gratch, Jina Lee, and Arno Hartholt. 2008. Multi-party, Multiissue, Multi-strategy Negotiation for Multi-modal Virtual Agents. In Proceedings of the 8th International Conference on Intelligent Virtual Agents, IVA '08, pages 117-130, Berlin, Heidelberg. SpringerVerlag.

Oriol Vinyals and Quoc Le. 2015. A Neural Conversational Model. arXiv preprint arXiv:1506.05869.

Tsung-Hsien Wen, David Vandyke, Nikola Mrksic, Milica Gasic, Lina M Rojas-Barahona, Pei-Hao Su, Stefan Ultes, and Steve Young. 2016. A Networkbased End-to-End Trainable Task-oriented Dialogue System. arXiv preprint arXiv:1604.04562.
Jason D Williams and Steve Young. 2007. Partially Observable Markov Decision Processes for Spoken Dialog Systems. Computer Speech \& Language, 21(2):393-422.

Ronald J Williams. 1992. Simple Statistical Gradientfollowing Algorithms for Connectionist Reinforcement Learning. Machine learning, 8(3-4):229-256. 